University of Wollongong

Research Online

Faculty of Engineering and Information

Faculty of Engineering and Information

Sciences - Papers: Part A

Sciences

2013

Self-adaptation-based dynamic coalition formation in a distributed agent network: a mechanism and a brief survey

Dayong Ye

University of Wollongong, dayong@uow.edu.au

Minjie Zhang

University of Wollongong, minjie@uow.edu.au

Danny Sutanto

University of Wollongong, soetanto@uow.edu.au

Follow this and additional works at: https://ro.uow.edu.au/eispapers

Part of the Engineering Commons, and the Science and Technology Studies Commons

Research Online is the open access institutional repository for the University of Wollongong. For further information contact the UOW Library: research-pubs@uow.edu.au 


\title{
Self-adaptation-based dynamic coalition formation in a distributed agent network: a mechanism and a brief survey
}

\begin{abstract}
In some real systems, e.g., distributed sensor networks, individual agents often need to form coalitions to accomplish complex tasks. Due to communication and computation constraints, it is infeasible for agents to directly interact with all other agents to form coalitions. Most previous coalition formation studies, however, overlooked this aspect. Those studies did not provide an explicitly modeled agent network or assumed that agents were in a fully connected network, where an agent can directly communicate with all other agents. Thus, to alleviate this problem, it is necessary to provide a neighborhood network structure, within which agents can directly interact only with their neighbors. Toward this end, in this paper, a self-adaptation-based dynamic coalition formation mechanism is proposed. The proposed mechanism operates in a neighborhood agent network. Based on selfadaptation principles, this mechanism enables agents to dynamically adjust their degrees of involvement in multiple coalitions and to join new coalitions at any time. The self-adaptation process, i.e., agents adjusting their degrees of involvement in multiple coalitions, is realized by exploiting a negotiation protocol. The proposed mechanism is evaluated through a comparison with a centralized mechanism (CM) and three other coalition formation mechanisms. Experimental results demonstrate the good performance of the proposed mechanism in terms of the entire network profit and time consumption. Additionally, a brief survey of current coalition formation research is also provided. From this survey, readers can have a general understanding of the focuses and progress of current research. This survey provides a classification of the primary emphasis of each related work in coalition formation, so readers can conveniently find the most related studies.
\end{abstract}

\section{Keywords}

era2015

Disciplines

Engineering | Science and Technology Studies

\section{Publication Details}

Ye, D., Zhang, M. \& Sutanto, D. (2013). Self-adaptation based dynamic coalition formation in a distributed agent network: a mechanism and a brief survey. IEEE Transactions on Parallel and Distributed Systems, 24 (5), 1042-1051. 


\title{
Self-Adaptation-Based Dynamic Coalition Formation in a Distributed Agent Network: A Mechanism and a Brief Survey
}

\author{
Dayong Ye, Minjie Zhang, Member, IEEE, and Danny Sutanto, Senior Member, IEEE
}

\begin{abstract}
In some real systems, e.g., distributed sensor networks, individual agents often need to form coalitions to accomplish complex tasks. Due to communication and computation constraints, it is infeasible for agents to directly interact with all other agents to form coalitions. Most previous coalition formation studies, however, overlooked this aspect. Those studies did not provide an explicitly modeled agent network or assumed that agents were in a fully connected network, where an agent can directly communicate with all other agents. Thus, to alleviate this problem, it is necessary to provide a neighborhood network structure, within which agents can directly interact only with their neighbors. Toward this end, in this paper, a self-adaptation-based dynamic coalition formation mechanism is proposed. The proposed mechanism operates in a neighborhood agent network. Based on self-adaptation principles, this mechanism enables agents to dynamically adjust their degrees of involvement in multiple coalitions and to join new coalitions at any time. The self-adaptation process, i.e., agents adjusting their degrees of involvement in multiple coalitions, is realized by exploiting a negotiation protocol. The proposed mechanism is evaluated through a comparison with a centralized mechanism (CM) and three other coalition formation mechanisms. Experimental results demonstrate the good performance of the proposed mechanism in terms of the entire network profit and time consumption. Additionally, a brief survey of current coalition formation research is also provided. From this survey, readers can have a general understanding of the focuses and progress of current research. This survey provides a classification of the primary emphasis of each related work in coalition formation, so readers can conveniently find the most related studies.
\end{abstract}

Index Terms-Distributed multiagent systems, coalition formation, self-adaptation

\section{INTRODUCTION}

A s agent technology becomes more capable and more A reliable, multiagent systems have been widely utilized to model real-world applications, such as distributed robotic systems [1], distributed sensor networks [2], supply chain management [3], cloud computing [4], and grid computing [5]. In many applications of multiagent systems, groups of agents need to dynamically join together in a coalition to complete a complex task, which none of them can complete independently. For example, in a distributed vehicletracking sensor network, to track a vehicle efficiently, at least three sensor agents are required to triangulate the position of a vehicle moving through the region [6].

Recently, many efforts have been done on coalition formation in multiagent systems, e.g., [7], [8], [9], [10]. There is a common assumption in these studies that an agent network structure is not explicitly modeled or such a structure is based on a fully connected network, namely that an agent can directly communicate with all the agents

- D. Ye and M. Zhang are with the School of Computer Science and Software Engineering, University of Wollongong, Wollongong NSW 2522, Australia.E-mail:dy721@uowmail.edu.au,minjie@uow.edu.au.

- D. Sutanto is with the School of Electrical, Computer and Telecommunications Engineering, University of Wollongong, Wollongong NSW 2522, Australia.E-mail: danny@elec.uow.edu.au.

Manuscript received 21 Sept. 2011; revised 24 June 2012; accepted 4 July 2012; published online 11 July 2012.

Recommended for acceptance by D. Turgut.

For information on obtaining reprints of this article, please send e-mail to: tpds@computer.org, and reference IEEECS Log Number TPDS-2011-12-0923. Digital Object Identifier no. 10.1109/TPDS.2012.213. in the network. ${ }^{1}$ However, in many real circumstances, particularly in large and distributed environments, it is infeasible for each individual agent to consider all the other agents to form coalitions due to time, communication and computation constraints, such as wireless sensor networks where each node has a limited communication range. To overcome this limitation, several researchers, e.g., [11], [12], imposed a neighborhood network structure among agents and required that agents directly communicate only with their neighbors. It should be noted that the introduction of such a network structure does not imply advantages or disadvantages. Instead, it just demonstrates the reality and constraints in the real world. For example, in some real systems, e.g., wireless sensor networks, due to the communication constraint, sensor nodes can directly communicate only with their neighbors, i.e., one hop nodes. Thus, in such sensor networks, if a node wants to form a coalition with other nodes to execute a task, e.g., several nodes collaboratively monitor a moving target, it is unlikely for the node to find coalition members directly in the whole sensor network but only from its neighbors (or neighbors' neighbors if necessary). Obviously, there are several new challenges, when coalition formation mechanisms are

1. Here, the phrase "network structure" denotes some sort of communication topologies of a network. If in a network, two agents can directly communicate with each other, they are considered to be a neighbor of each other, i.e., neighborhood. "Neighborhood network structure" has the same meaning with "network structure" in this paper. An "agent network" indicates a network, which is connected by a number of agents and which is formed in a communication topology as well. 
designed in a structured agent network, such as direct communication of agents with their neighbors to find potential coalition members and uncertainty of distant activities. Gaston and desJardins [11], [13], and Glinton et al. [12] have made great efforts in this way. They investigated the impact of diverse network structures on coalition formation among agents and found that an underlying network structure does have a crucial effect on the performance of agents coalition formation. The studies done in [11], [13], and [12] initiated a new research field in the study of coalition formation, i.e., designing coalition formation mechanisms within explicitly modeled network structures. However, since their research focused on the effect of network structures on coalition formation, the coalition formation mechanisms developed in their research were relatively simple. In their mechanisms [11], [13], [12], an agent can join only one coalition and once a coalition is formed for a task, the coalition is fixed and agents cannot leave the coalition before the task is finished.

Against this background, in this paper, we design a coalition formation mechanism in a structured agent network, which is claimed as a main contribution of this paper. The proposed mechanism assumes that there is a network with explicit links between the agents, such that only agents that are linked to each other (directly or indirectly) can form coalitions. Additionally, our coalition formation mechanism incorporates the self-adaptation concept, which enables agents to dynamically adjust their degrees of involvement in coalitions and to join new coalitions, via negotiation, at any time if necessary. The process of self-adaptation in a large-scale and distributed system is of key importance to the performance of the system as a whole and it can be employed in agent networks to improve the cooperative behaviors of agents [14]. Compared with most related studies, which do not take a network structure into account, we consider the existence of an underlying network structure. Compared with those related studies, which do consider network structures, our mechanism, by integrating the self-adaptation notion, enables agents to have autonomy and flexibility when agents execute tasks. Our mechanism is elucidated by using distributed task allocation. By employing a general application area, i.e., distributed task allocation, instead of a particular existing system, we can develop a general mechanism that can be potentially applied to a wide variety of applications.

The remainder of the paper is organized as follows: Section 2 introduces the agent network model. Section 3 proposes the dynamic coalition formation mechanism. Experimental results and analysis are presented in Section 4 and the paper is concluded in Section 5. The brief survey of current coalition formation studies and the discussion about the difference between our work and these studies are given in the supplementary file, which can be found on the Computer Society Digital Library at http://doi. ieeecomputersociety.org/10.1109/TPDS.2012.213.

\section{Agent Network Model}

To cope with the issue of coalition formation in a structured agent network, we first give the formal definition of the agent network.
Definition 1. (Agent Network). An agent network consists of a set of interdependent agents, namely, $A=\left\{a_{1}, \ldots, a_{n}\right\}$, and a Compatible Relation $R, R \subseteq A \times A$. The meaning of $R$ is "a neighbor of," so we denote an ordered couple $\left\langle a_{i}, a_{j}\right\rangle \in R$ if and only if $a_{j}$ is a neighbor of $a_{i}$. Since $R$ is a Compatible Relation, namely that $R$ is reflexive and symmetric, it can be achieved that $\forall a_{i}: a_{i} \in A \Rightarrow\left\langle a_{i}, a_{i}\right\rangle \in R$ and $\forall a_{i}, a_{j} \in$ $A:\left\langle a_{i}, a_{j}\right\rangle \in R \Rightarrow\left\langle a_{j}, a_{i}\right\rangle \in R$.

The definition of the reflexive and symmetric relation $R$ is to indicate that the connection between two agents is bidirectional. This definition is necessary, because when an agent wants another agent to join the former's coalition, there is a negotiation between them (described in Section 3).

Each agent $a \in A$ is composed of three tuples $\left\langle r_{a}, N e i g(a)\right.$, State $\left.(a)\right\rangle$, where, $r_{a}$ is the resource, which agent a contains, $N \operatorname{eig}(a)$ is a set, which contains the neighbors of agent $a$, and State $(a)$ demonstrates the state of agent $a$. Each agent $a \in A$ has a single fixed resource, ${ }^{2} r_{a} \in[1, \varepsilon]$, where $\varepsilon$ is the number of different resources that are present in the agent network. Before describing the states of an agent, we provide the definition of three roles used throughout this paper, i.e., Initiator, Participant, and Mediator.

Definition 2. ((Initiator), (Participant), and (Mediator)). In an agent network, the agent, which initializes a task allocation is called Initiator; the agent, which accepts the announced task is called Participant; and the agent that receives another agent's commitments for assistance to find Participants is called Mediator.

It should be noted that the roles, i.e., Initiator, Participant, and Mediator, do not imply any architecture in a coalition. Instead, different roles are used only to distinguish different responsibilities of agents.

Definition 3. (States). There are two states of agents in the network, i.e., States $=\{I D L E, B U S Y\}$, and an agent can be only in one of the two states at any time step. When an agent is an Initiator, Participant, or Mediator the state of an agent is BUSY. An agent in IDLE state is available and has not been assigned or committed to any task.

In this paper, it is supposed that only an IDLE agent can be assigned to a new task as an Initiator; both IDLE and BUSY agents can join partially fulfilled tasks as Participants or be committed to partially fulfilled tasks as Mediators. A partially fulfilled task is a task, for which a full coalition is in the formation procedure and has not yet formed.

Suppose there is a set of tasks $\Theta=\left\{\theta_{1}, \ldots, \theta_{m}\right\}$ arriving at the agent network. Each task $\theta \in \Theta$ consists of four tuples, namely $\langle A T(\theta), D L(\theta), P D(\theta), R(\theta)\rangle . A T(\theta)$ is the arrival time of task $\theta, D L(\theta)$ indicates the latest start time of task $\theta$, $P D(\theta)$ indicates that how long it will take to finish the task $\theta$ and $R(\theta)=\left\{r_{\theta}^{1}, \ldots, r_{\theta}^{j}\right\}$ is a set that dictates the resources, which are needed for successfully completing the task. Therefore, for each task, the Initiator has to find appropriate agents, which have the required resources, to form a coalition to handle the task. The term coalition is defined as follows.

2. This assumption will be relaxed in Section 4 . 
Definition 4. (Coalition). A coalition $c$ is a set of agents, i.e., $c \subseteq A$, which cooperate together to complete a complex task $\theta \in \Theta$. Each coalition is associated with a task, and then there is a set of coalitions $C=\left\{c_{1}, \ldots, c_{h}\right\}$, which are associated with tasks, $\theta_{1}, \ldots, \theta_{h}$, respectively. A valid coalition should satisfy the situation that the resources of agents in the coalition should cover the required resources of the associated task, i.e., $\bigcup_{a \in c_{j}} r_{a} \supseteq R\left(\theta_{j}\right)$.

To form a coalition, the Initiator of a task needs to make a contract with each Participant. The details of contracts will be described later in Section 3.1. It should be noted that, in this paper, each agent is allowed to join multiple coalitions. Thus, the term, "degree of involvement," is also needed.

Definition 5. (Degree of Involvement (DoI) $\left.{ }^{3}\right)$. A degree of involvement indicates the extent that an agent belongs to a coalition. The value of DoI is ranged in $(0,1]$, where a higher value means that an agent belongs to a coalition in a larger extent. The sum of DoI values of an agent in different coalitions should be equal to or less than 1.

A DoI in a coalition has an effect on the cost of an agent to complete a task. The initial DoI value of an agent joining a coalition is determined by a contract, which is achieved through negotiation between the Initiator and the Participant. The DoI value of an agent can be adjusted at any time in the future by paying penalty to the counterpart. Thus, it can be seen that a coalition is formed via making contracts between the Initiator and Participants.

After formalizing the agent network, the principle of our coalition formation mechanism will be depicted.

\section{Coalition Formation Mechanism}

In an agent network, agents make decisions based only on local information about the system, and the decision making process of agents is autonomous without external control. Hence, we need to define another set $P=\left\{P_{1}, \ldots, P_{n}\right\}$. $P$ is defined as a partition of the Compatible Relation $R$, which has been described in Definition 1. Accordingly, it can be obtained that $\bigcup_{1 \leq i \leq n} P_{i}=R$ and $\forall P_{i}, P_{j} \in P: i \neq j \Rightarrow P_{i} \cap P_{j}=\emptyset$. The set $P$ can be generated by using Algorithm 1 .

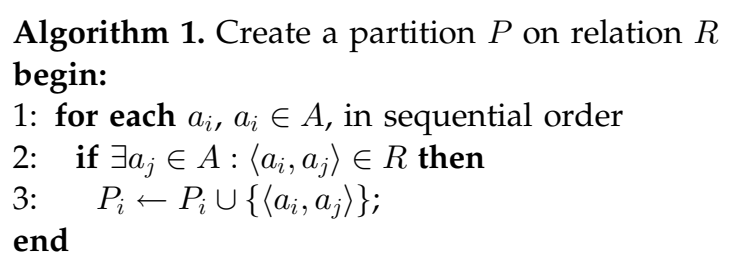

From Algorithm 1, it can be found that $P_{i}$ is composed of ordered couples, and each ordered couple dictates an agent, which is a neighbor of $a_{i} . P_{i}$ and $\operatorname{Neig}\left(a_{i}\right)$ look like having the same meaning, but, actually, they are used for different purposes. $P_{i}$ represents not only neighbors of agent $a_{i}$ but also other agents, which have indirect connections with $a_{i}$

3. The concept of "degree of involvement" was originally mentioned in operation research field, e.g., [15], [16], where such a concept described the participation level of a person to an organization, e.g., how much time or how many resources a person uses in an organization. In this paper, this concept is borrowed for our problem. established during future task allocation processes, although $P_{i}$ initially denotes only neighbors of agent $a_{i}$. On the other hand, Neig $\left(a_{i}\right)$ stores only directly linked neighboring agents of agent $a_{i}$. The coalition formation mechanism, used by an Initiator to find appropriate agents to form a coalition, is illustrated in Algorithm 2 as follows.

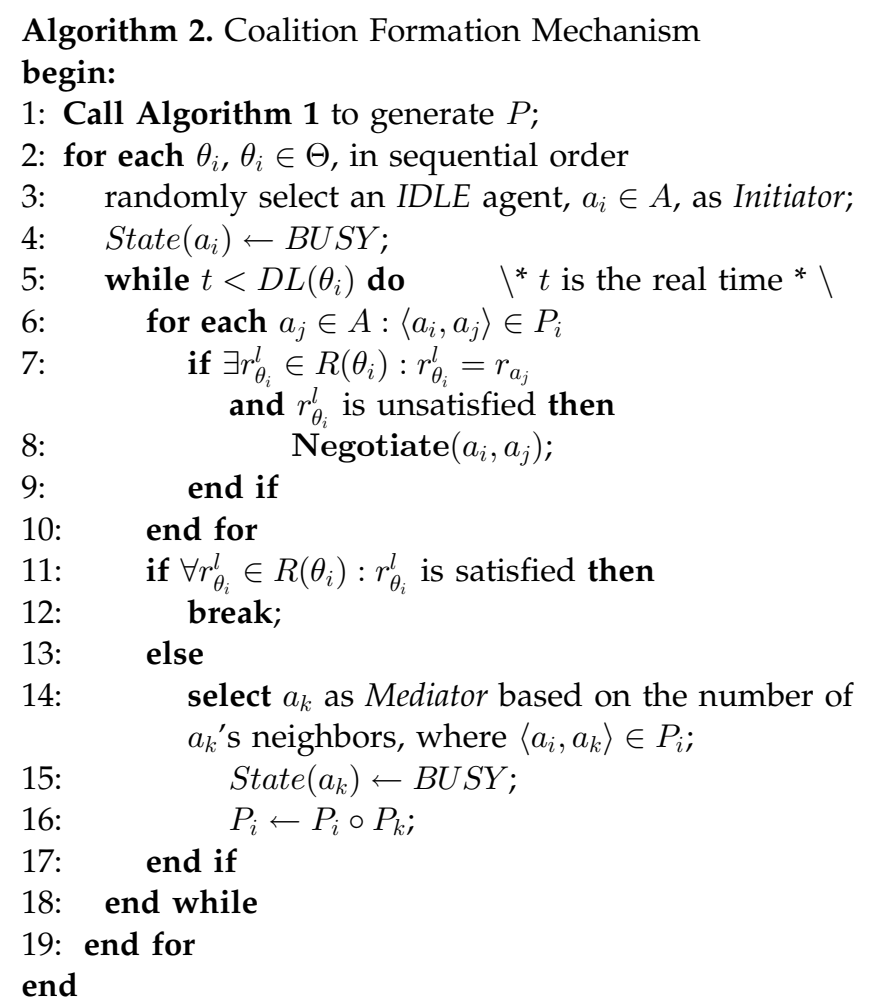

For each task $\theta_{i}$, in Line 3 , an IDLE agent is randomly selected to be an Initiator, denoted as $a_{i}$. In this paper, tasks come from outside of the agent network. A new task can arrive at each time step with a specific probability, and is given to a randomly selected IDLE agent. Then, before the deadline of task $\theta_{i}$ (Line 5), $a_{i}$ checks whether the resources of its neighbors, including itself, can satisfy any resource requirements of task $\theta_{i}$. If any neighboring agent can satisfy any one of the resource requirements, $a_{i}$ will negotiate with this agent regarding the DoI of this agent in $a_{i}$ 's coalition (Lines 6-10). The negotiation protocol (Line 8), adopted to make a contract between an Initiator and a Participant, will be described later. It should be noted that, in Line $6, a_{i}$ selects coalition members only from the set $P_{i}$, which consists of $a_{i}{ }^{\prime} \mathrm{s}$ neighbors (and mediators' neighbors if any). Thus, our coalition formation mechanism takes the communication constraint into account. Thereafter, in Lines 11-13, if all the resource requirements of task $\theta_{i}$ are satisfied, the coalition has been formed and the task can be started. It should be noted that, for a single resource requirement, $a_{i}$ has to make several temporary agreements, i.e., temporary contracts, with several different agents, (one temporary agreement with one distinct agent), because agents may cancel temporary agreements before final agreements are made and leave the Initiator $a_{i}$ very little time to find an alternative. Thus, multiple temporary agreements are used to reduce the possibility of an Initiator's failure to form a coalition. However, making too many temporary agreements for a single resource will incur much communication cost to the 
Initiator. Hence, there is a tradeoff with regard to the number of temporary agreements for a single resource. In this paper, the number of temporary agreements for a single resource is set to two, which is obtained through experiments to achieve the best results. Here, a temporary agreement means that when an agent, say $a_{j}$, decides to join the Initiator $a_{i}$ 's partial formed coalition, where some task resource requirements have not been satisfied, the agreement between $a_{i}$ and $a_{j}$ is a temporary agreement. A temporary agreement can be canceled by either the Initiator or the Participant at any time without paying penalty. Once the coalition becomes a full coalition, namely that all the task resource requirements are satisfied, those uncanceled temporary agreements will automatically become final agreements and cannot be canceled by either party of the agreements. For the clarity purpose, such details of temporary agreements are not demonstrated in Algorithm 2. On the other hand, if any resource requirement is not satisfied, in Line $14, a_{i}$ selects one of the agents in the set $P_{i}$ as Mediator, say $a_{k}$, and commits task $\theta_{i}$ to $a_{k}$. Then, $a_{k}$ will be responsible for $a_{i}$ to find available coalition members from $a_{k}$ 's neighbors. The Mediator selection is based on the number of neighbors that each neighbor of $a_{i}$ has. The more neighbors an agent has, the higher probability that agent could be selected as a Mediator. In the case that $a_{k}$ 's neighbors still cannot fulfill the resource requirements of task $\theta_{i}, a_{k}$ chooses one of its neighbors as a new Mediator and commits task $\theta_{i}$ to the new Mediator. This process will continue until the deadline of task $\theta_{i}$ is reached. In Line 16, the notation "o" is relational composition. The meaning of this notation is that $\forall\left\langle x_{i}, y_{i}\right\rangle \in$ $X,\left\langle y_{j}, z_{j}\right\rangle \in Y: y_{i}=y_{j} \Rightarrow\left\langle x_{i}, z_{j}\right\rangle \in Z$. In this paper, relational composition is utilized to model indirect connections between Initiators and Participants via Mediators. Through this way, an Initiator can request not only its directly linked neighbors but also other indirectly connected agents for help.

\subsection{The Negotiation Protocol}

To operate the coalition formation mechanism, we need another important component, i.e., a negotiation protocol. The coalition formation problem can be modeled as a negotiation process between an Initiator and a Participant, where an Initiator acts as a buyer and a Participant plays as a seller. The negotiation focuses on a single issue, i.e., the DoI of a Participant into a coalition, which is being formed by an Initiator. Two constraints are listed as follows, with which each agent should comply:

1. The DoI of an Initiator in its initiated coalition is postulated to be 1 and cannot be adapted. ${ }^{4}$ Other agents, which are not Initiators, can dynamically join multiple coalitions with different degrees of involvement.

2. Both Initiators and Participants cannot cancel final agreements. Participants can adapt the degrees of involvement in their joined coalitions by paying penalty to Initiators, and Participants can join other new coalitions if necessary. Temporary agreements

4. This postulation is set, because it is conceived that an Initiator usually has to negotiate with many other agents to form a coalition, and thus, it usually does not have enough energy and time to finish other Initiators' tasks. can be canceled by either Initiators or Participants without paying penalty.

The negotiation protocol employed in this paper extends the alternating offers protocol [17] by allowing an agent to make multiple agreements with other agents and to cancel temporary agreements without paying penalty. The alternating offers protocol [17] has been widely used for bilateral bargaining (e.g., An et al. [18]). Other more complex negotiation protocols may be also available for our problem, e.g., [19], [20], but based on our investigation, the alternating offers protocol is powerful enough for our problem and it is easy to implement. It should be noted that the main contribution of this paper is the idea of integrating the selfadaptation notion into dynamic coalition formation rather than this negotiation protocol used only for realizing the self-adaptation notion.

There are four possible actions that a buyer (Initiator) and a seller (Participant) can take are as follows:

- offer $[o]$, where $o$ is buyer's offer to a seller. An offer is determined by four factors, which are the pressure of deadline, the payment of the resource paid by the buyer to the seller, the duration of using the resource, and the demand/supply ratio of the buyer's required resource.

- accept $[o]$. When a seller receives an offer $o$, it can accept the offer, which results in a temporary agreement made with the buyer.

- counter_offer $\left[o^{\prime}\right]$. If a seller is not happy with an offer $o$, it can send back a counter-offer $o^{\prime}$ for its available resource. A counter-offer $o^{\prime}$ is determined by three aspects, which include the current state of the seller, e.g., whether it has joined other coalitions and the degrees of involvement into those coalitions, the payment received by the seller from the buyer, and the demand/supply ratio of the seller's available resource.

- cancel[o]. After a temporary agreement is achieved by a buyer and a seller, any one of them can cancel the temporary agreement without paying penalty. A final agreement, however, cannot be canceled by either a buyer or a seller.

The negotiation protocol, displayed in Line 8 of Algorithm 2, is shown in Algorithm 3 as follows.

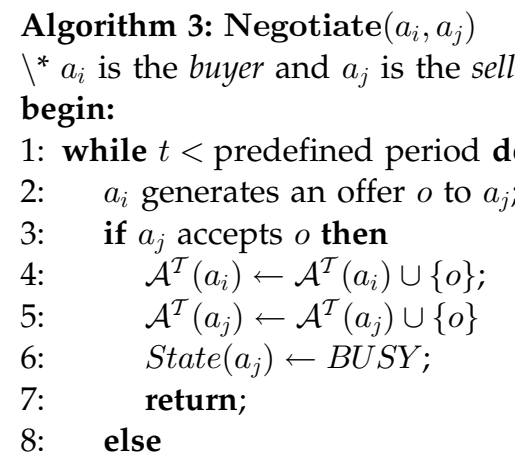

$a_{j}$ generates a counter-offer $o^{\prime}$ to $a_{i}$;

if $a_{i}$ accepts $o^{\prime}$ then

$$
\mathcal{A}^{\mathcal{T}}\left(a_{i}\right) \leftarrow \mathcal{A}^{\mathcal{T}}\left(a_{i}\right) \cup\left\{o^{\prime}\right\} ;
$$$$
\mathcal{A}^{\mathcal{T}}\left(a_{j}\right) \leftarrow \mathcal{A}^{\mathcal{T}}\left(a_{j}\right) \cup\left\{o^{\prime}\right\} ;
$$ 


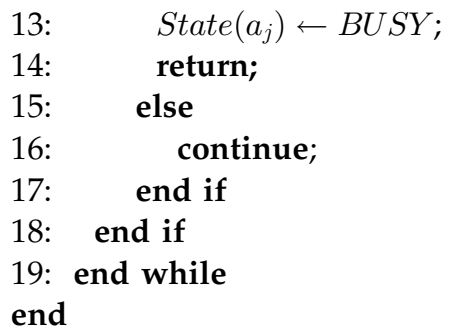

During a predefined negotiation period, in Line 2, the buyer $a_{i}$ first generates an offer $o$ and sends the offer to the seller $a_{j}$, where $o=\left\langle p a y, D o I_{u}, D o I_{l}, p e, p d\right\rangle$. Each element in $o$ is calculated by using the following equations:

$$
\begin{aligned}
& \text { pay }= \begin{cases}p\left(r_{a_{j}}\right) \frac{t-A T(\theta)}{D L(\theta)-A T(\theta)}, & \text { if } \mathcal{A}^{\mathcal{T}}\left(a_{i}\right)=\emptyset, \\
\frac{p\left(r_{a_{j}}\right)}{\left|\mathcal{A}^{\mathcal{T}}\left(a_{i}\right)\right|} \frac{t-A T(\theta)}{D L(\theta)-A T(\theta)}, & \text { otherwise, }\end{cases} \\
& D o I_{u}=1-\frac{p a y}{p\left(r_{a_{j}}\right)}, \\
& D o I_{l}=\frac{D o I_{u}}{\tau}, \\
& p e=\alpha \cdot p a y, \\
& p d=P D(\theta) .
\end{aligned}
$$

In (1), pay is the intended payment made by $a_{i}$ to $a_{j}$, $p\left(r_{a_{j}}\right)$ is the maximum payment that $a_{i}$ can endure for the required resource $r_{a_{j}}, \mathcal{A}^{\mathcal{T}}\left(a_{i}\right)$ is the set of temporary agreements achieved by $a_{i}$ for the resource $r_{a_{j}}$, and $\left|\mathcal{A}^{\mathcal{T}}\left(a_{i}\right)\right|$ denotes the number of temporary agreements in the set $\mathcal{A}^{\mathcal{T}}\left(a_{i}\right)$. An Initiator can get benefit from the task if and only if all the subtasks of the task are completed, while a Participant can obtain the relevant payment when it finishes the assigned subtask. Here, a subtask corresponds to a resource requirement of a task.

In (2), DoI $I_{u}$ is the upper bound DoI in the coalition, which $a_{i}$ wants $a_{j}$ to join for task $\theta$. It can be found that $a_{i}{ }^{\prime} \mathrm{s}$ expected $D o I_{u}$ value of $a_{j}$ in $a_{i}$ 's coalition decreases with the increase of payment from $a_{i}$ to $a_{j}$, which seems somewhat weird. This is caused by $a_{i}{ }^{\prime} \mathrm{s}$ concession strategy, as $a_{i}{ }^{\prime} \mathrm{s}$ deadline is approaching. Such time-dependent concession strategies have been broadly used in the literature (e.g., Faratin et al. [21]).

In (3), $D o I_{l}$ is the lower bound DoI about $a_{j}$ in $a_{i}{ }^{\prime} \mathrm{s}$ coalition, which means that $a_{j}$ cannot reduce its $D o I$ value in $a_{i}$ 's coalition less than $D o I_{l} . \tau$ is a coefficient, which is a positive integer. Thus, if $a_{j}$ accepts an offer from $a_{i}$, its original DoI in $a_{i}{ }^{\prime}$ coalition is $D o I_{u}$. $a_{j}$ is able to decrease its $D o I$ value in $a_{i}{ }^{\prime}$ coalition later, but the $D o I$ value must not be less than $D o I_{l}$.

In (4), $\alpha$, where $0<\alpha<1$, is a coefficient and pe is the total penalty if $a_{j}$ wants to reduce the DoI in $a_{i}{ }^{\prime}$ s coalition from the upper bound to the lower bound, i.e., from $D o I_{u}$ to $D o I_{l}$. The exact penalty $a_{j}$ should pay to $a_{i}$, recorded as $p e_{j \rightarrow i}$, is based on the extent that $a_{j}$ wants to lessen the DoI in $a_{i}{ }^{\prime}$ s coalition. Specifically, $p e_{j \rightarrow i}$ can be calculated by using (6), where DoI' is the current DoI and DoI" is the intended DoI to which $a_{j}$ wants to adjust. For example, $a_{j}{ }^{\prime} \mathrm{s}$ original $D o I$ value in $a_{i}{ }^{\prime}$ s coalition is 1 , i.e., $D o I_{u}=1$ and the lower bound is 0.33, i.e., $D o I_{l}=0.33$ (suppose $\tau=3$ ). Its current $D o I$ value in $a_{i}{ }^{\prime}$ s coalition is 0.8 , i.e., $D o I^{\prime}=0.8$ (suppose that $a_{j}$ has changed its $D o I$ value in $a_{i}$ 's coalition to join another coalition with $D o I$ value 0.2 ). Now, an agent, say $a_{k}$, also wants $a_{j}$ to join its coalition with $D o I$ value 0.3 . Then, $a_{j}$ may reduce the $D o I$ value in $a_{i}$ 's coalition again from 0.8 to 0.5 , i.e., $D o I^{\prime \prime}=0.5$, by paying the penalty, $p e_{j \rightarrow i}=\frac{p e}{1-0.33} \cdot(0.8-0.5)$, to $a_{i}$, and simultaneously join $a_{k}$ 's coalition with $D o I$ value 0.3 , (recall that the sum of $D o I$ values of $a_{j}$ should not be greater than 1). $a_{j}$ never wants to increase its $D o I$ value in any coalition, as this will not bring any revenue to $a_{j}$ but will even incur an extra cost to $a_{j}$

$$
p e_{j \rightarrow i}=\frac{p e}{D o I_{u}-D o I_{l}} \cdot\left(D o I^{\prime}-D o I^{\prime \prime}\right) .
$$

In (5), $p d$ indicates the period, during which the required resource is needed.

After receiving an offer $o$ from $a_{i}$, in Line 3, $a_{j}$ evaluates whether the offer $o$ is acceptable. This evaluation is based on how much revenue $a_{j}$ could get (7). In (7), the cost of $a_{j}$ depends on its DoI in $a_{i}$ 's coalition and how long its resource will be used by $a_{i}$. The notation $p e_{j \rightarrow k}$ means the penalty that $a_{j}$ has to pay other Initiators if $a_{j}$ wants to lower its $D o I$ values in their coalitions.

$$
r v=\left\{\begin{array}{lr}
\text { pay }- \text { cost }, & \text { if } \mathcal{A}^{\mathcal{T}}\left(a_{j}\right)=\emptyset, \\
\text { pay }- \text { cost }-p e_{j \rightarrow k}, & \text { otherwise }
\end{array}\right.
$$

If $r v$ is greater than a predefined threshold, $a_{j}$ will accept the offer $o$ and a temporary agreement is achieved (Lines 4 and 5). Otherwise, $a_{j}$ generates a counter-offer $o^{\prime}$ to $a_{i}$ (Line 9). The elements, which consist of a counter-offer $o^{\prime}$, are the same as those in an offer $o$. Since the negotiation issue is only the DoI as described earlier, $a_{j}$ will adjust only its $D o I$ value in $o$ to meet its predefined threshold revenue via (7) and will create a counter-offer $o^{\prime}$ with the newly calculated $D o I$ value. $a_{i}$ then will evaluate the counter-offer $o^{\prime}$ by comparing the $D o I$ value in $o^{\prime}$ with its reserved $D o I$ value. If the $D o I$ value in $o^{\prime}$ is greater than its reserved $D o I$ value, $o^{\prime}$ is acceptable and a temporary agreement is achieved (Lines 11 and 12). Otherwise, $a_{i}$ continues to go to the next negotiation round (Line 16) until the predefined negotiation period is reached.

When all the resource requirements are satisfied, the Initiator, i.e., the buyer, $a_{i}$ will select the most valuable (the least payment) temporary agreement and cancel other temporary agreements. For the Participant, i.e., the seller, $a_{j}$ can execute several tasks simultaneously with the summation of $D o I$ values equal to or less than 1.

\subsection{How Does an Agent Adjust Dol Values?}

There is another problem in the coalition formation process, which has to be solved, i.e., how can an agent adjust its $D o I$ values in current coalitions to minimize its penalty when it joins a new coalition? Here is an example to illustrate the problem. An agent has joined three coalitions with DoI values $0.2,0.3$, and 0.5 , respectively. After negotiation, this agent decides to join a new coalition with $D o I$ value 0.4 . Now, the agent has to decide how to adjust the $D o I$ values 
in the three current coalitions, as the sum of the $D o I$ values in the current three coalitions should be at most 0.6 (derived from $1-0.4$ ) and this agent has to minimize its penalty (remember that reducing $D o I$ value in a coalition will incur penalty). We first formulate the problem and then provide an algorithm to solve this problem.

Let us suppose that an agent, say $a_{j}$, has joined $n$ coalitions. The sum of $D o I$ values in these $n$ coalitions is 1 , i.e., $\sum_{1 \leq i \leq n} D o I_{i}=1$. Now another agent also wants $a_{j}$ to join its coalition with the DoI $D o I_{n+1}$. If $a_{j}$ is interested in the new offer, i.e., $D o I_{n+1}$, it has to calculate the minimal penalty it will pay to the $n$ coalition leaders. It is assumed that $a_{j}$ reduces $\Delta D o I_{i}$ in $i$ th coalition, where $0 \leq \Delta D o I_{i} \leq$ $D o I_{i}-D o I_{l}^{i}$. The question for $a_{j}$ is how to select each $\Delta D o I_{i}$ to minimize $\sum_{1 \leq i \leq n} \frac{p e_{i}}{D o I_{u}^{i}-D o I_{i}^{i}} \Delta D o I_{i}$, given $\sum_{1 \leq i \leq n}\left(D o I_{i}-\right.$ $\left.\Delta D o I_{i}\right)+D o I_{n+1}=1$, where $p e_{i}$ is the total penalty if $a_{j}$ wants to reduce the DoI in $a_{i}{ }^{\prime}$ s coalition from the upper bound to the lower bound. For convenience, we denote peRate $_{i}=\frac{p e_{i}}{D o I_{u}^{i}-D o I_{i}^{2}}$, whose value is known by $a_{j}$. Likewise, the given condition can be written as another form $\sum_{1 \leq i \leq n} \Delta D o I_{i}=C$ and $C=\sum_{1 \leq i \leq n+1} D o I_{i}-1$. Since each $D o I_{i}(1 \leq i \leq n+1)$ is known by $a_{j}$, the value of $C$ is known by $a_{j}$ as well. The question now can be represented as how to select each $\Delta D o I_{i}$ to minimize $\sum_{1 \leq i \leq n}$ peRate $_{i} \cdot \Delta D o I_{i}$, given $\sum_{1 \leq i \leq n} \Delta D o I_{i}=C$. Algorithm 4 can be used to derive the optimal $\Delta D o I_{i}$.

\section{Algorithm 4: Adjusting $D o I$ values}

$\backslash$ Suppose that agent $a_{j}$ attempts to adjust $n D o I$ values * begin:

1: if $\sum_{1 \leq i \leq n}\left(D o I_{i}-D o I_{l}^{i}\right)<C$ then

2: break; $\backslash^{*}$ no solution in this case, the offer is unacceptable * $\backslash$

3: else if $\sum_{1 \leq i \leq n}\left(D o I_{i}-D o I_{l}^{i}\right)=C$ then

4: for each $\Delta D o I_{i} ; \backslash^{*} 1 \leq i \leq n^{*} \backslash$

5: $\quad \Delta D o I_{i} \leftarrow D o I_{i}-D_{0} I_{l}^{i}$;

6: else $\backslash * \sum_{1 \leq i \leq n}\left(D o I_{i}-D o I_{l}^{i}\right)>C^{*} \backslash$

7: $\quad$ ranking peRate , $_{i}$ such that peRate $1 \leq \cdots \leq$ peRate $_{n}$;

8: $\quad$ find an integer $k, 1 \leq k<n$, such that

9: $\quad \begin{aligned} & \sum_{1 \leq i<k}\left(D o I_{i}-D o I_{l}^{i}\right)<C \text { and } \\ & \quad \sum_{1 \leq i \leq k}\left(D o I_{i}-D o I_{l}^{i}\right) \geq C ;\end{aligned}$

10: $\quad$ for $1 \leq i<k$

11: $\quad \Delta D o I_{i} \leftarrow D o I_{i}-D o I_{l}^{i}$;

12: $\quad$ for $i=k$

13: $\Delta D o I_{i} \leftarrow C-\sum_{1 \leq i<k}\left(D o I_{i}-D o I_{l}^{i}\right)$;

14: $\quad$ for $k<i \leq n$

15: $\Delta D o I_{i} \leftarrow 0$;

16: end if

end

The proof of validity of Algorithm 4 is provided as follows.

Proposition 3.1. The result derived from Algorithm 4 can minimize $\sum_{1 \leq i \leq n}$ peRate $\cdot \cdot \Delta$ DoI $_{i}$, given $\sum_{1 \leq i \leq n} \Delta D o I_{i}=C$.

Proof. For cases 1 and 2, i.e., $\sum_{1 \leq i \leq n}\left(D o I_{i}-D o I_{l}^{i}\right)<C$ and $\sum_{1 \leq i \leq n}\left(D o I_{i}-D o I_{l}^{i}\right)=C$, the validity of the algorithm is obvious. Thus, our proof focuses on case 3, i.e., $\sum_{1 \leq i \leq n}\left(D o I_{i}-D o I_{l}^{i}\right)>C$.
According to Algorithm 4, in case 3, it is easy to deduce that $\sum_{1 \leq i \leq n} \Delta D o I_{i}=C$, so the condition can be satisfied. We arbitrarily select $\Delta D o I_{1}^{\prime}, \ldots, \Delta D o I_{n}^{\prime}$, where each $\Delta D o I_{i}^{\prime} \in\left[0, D o I_{i}-D o I_{l}^{i}\right]$ and $\sum_{1 \leq i \leq n} \Delta D o I_{i}^{\prime}=C$. What we need to do now is to prove $\sum_{1 \leq i \leq n}$ peRate $e_{i} \cdot \Delta D o I_{i}^{\prime} \geq$ $\sum_{1 \leq i \leq n}$ peRate $_{i} \cdot \Delta D o I_{i}$. It is easy to achieve that

$$
\left\{\begin{array}{l}
\Delta D o I_{i}^{\prime} \leq \Delta D o I_{i}=D o I_{i}-D o I_{l}^{i}, 1 \leq i<k \\
\Delta D o I_{i}^{\prime} \geq \Delta D o I_{i}=0, k<i \leq n .
\end{array}\right.
$$

If $\Delta D o I_{k}^{\prime} \geq \Delta D o I_{k},{ }^{5}$ because $\sum_{1 \leq i \leq n}\left(\Delta D o I_{k}^{\prime}-\Delta\right.$ $\left.D o I_{k}\right)=\sum_{1 \leq i \leq n} \Delta D o I_{k}^{\prime}-\sum_{1 \leq i \leq n} \Delta D o I_{k} \stackrel{i \leq n}{=} C-C=0$, we, thus, have $\sum_{1 \leq i<k}-\left(\Delta D o I_{k}^{\prime}-\Delta D o I_{k}\right)=\sum_{k \leq i \leq n}(\Delta$ DoI $_{k}^{\prime}-\Delta$ DoI $_{k}$ ). Therefore, from peRate ${ }_{1} \leq \cdots \leq$ peRate $_{n}$, we can deduce that

$$
\begin{aligned}
& \sum_{1 \leq i \leq n} \text { peRate }_{i} \cdot \Delta D o I_{i}^{\prime}-\sum_{1 \leq i \leq n} \text { peRate }_{i} \cdot \Delta D o I_{i} \\
& =\sum_{1 \leq i \leq n} \text { peRate }_{i} \cdot\left(\Delta D o I_{k}^{\prime}-\Delta D o I_{k}\right) \\
& =\sum_{1 \leq i<k}-\text { peRate }_{i} \cdot-\left(\Delta D o I_{k}^{\prime}-\Delta D o I_{k}\right) \\
& +\sum_{k \leq i \leq n} \text { peRate }_{i} \cdot\left(\Delta D o I_{k}^{\prime}-\Delta D o I_{k}\right) \\
& \geq \sum_{1 \leq i<k}-\text { peRate }_{k-1} \cdot-\left(\Delta \text { DoI }_{k}^{\prime}-\Delta D_{o} I_{k}\right) \\
& +\sum_{k \leq i \leq n} \text { peRate }_{k} \cdot\left(\Delta D o I_{k}^{\prime}-\Delta D o I_{k}\right) \\
& =- \text { peRate }_{k-1} \cdot \sum_{1 \leq i<k}-\left(\Delta D o I_{k}^{\prime}-\Delta D o I_{k}\right) \\
& + \text { peRate }_{k} \cdot \sum_{k \leq i \leq n}\left(\Delta D o I_{k}^{\prime}-\Delta D o I_{k}\right) \\
& =- \text { peRate }_{k-1} \cdot \sum_{k \leq i \leq n}\left(\Delta D o I_{k}^{\prime}-\Delta D o I_{k}\right) \\
& + \text { peRate }_{k} \cdot \sum_{k \leq i \leq n}\left(\Delta D o I_{k}^{\prime}-\Delta D o I_{k}\right) \\
& =\left(\text { peRate }_{k}-\text { peRate }_{k-1}\right) \cdot \sum_{k \leq i \leq n}\left(\Delta \text { DoI I }_{k}^{\prime}-\Delta D o I_{k}\right) \geq 0
\end{aligned}
$$

Then, this result indicates that $\Delta D o I_{1}, \ldots, \Delta D o I_{n}$ derived from Algorithm 4 can minimize $\sum_{1 \leq i \leq n}$ peRate ${ }_{i}$. $\Delta D o I_{i}$, given $\sum_{1 \leq i \leq n} \Delta D o I_{i}=C$.

After illustrating our self-adaptation-based coalition formation mechanism, in the next section, we will demonstrate its performance in a simulated agent network in comparison with other coalition formation mechanisms.

\section{EXPERIMENTS AND ANALYSIS}

To evaluate the performance of the proposed coalition formation mechanism, a set of simulation experiments are conducted. In these experiments, an agent network is initialized by using the random geometric graphs [22], which can be constructed to have a wide range of densities, i.e., the varying average number of neighbors. We first describe the experimental setup and thereafter present the experimental results and analysis.

5. For the case that $\Delta D o I_{k}^{\prime}<\Delta D o I_{k}$, the deducing process is the same. 
TABLE 1

Parameters Setting

\begin{tabular}{|c|c|c|}
\hline Para. & Values & Explanations \\
\hline$n$ & $200 \sim 400$ & The number of agents \\
\hline$\epsilon$ & 10 & The number of resource types \\
\hline deg & $4 \sim 12$ & The number of time steps \\
\hline$m$ & 50000 & The ratio to calculate penalty from payment \\
\hline$\alpha$ & 0.2 & The ratio of upper bound to lower bound $D o I$ values \\
\hline$\tau$ & 3 & The probability with which tasks are introduced \\
\hline$\lambda$ & $0.1 \sim 0.9$ &
\end{tabular}

\subsection{Experimental Setup}

To objectively exhibit the effectiveness of our coalition formation mechanism, named self-adaptation coalition formation $(S A C F)$, we compare our mechanism with three other mechanisms, i.e., Centralized mechanism (CM), classic coalition formation (CCF) [12] mechanism, and flexible coalition formation $(F C F)$ mechanism.

1. CM. This is an ideal centralized coalition formation mechanism, in which there is an external omniscient central manager that maintains information about all the agents and tasks. The central manager is able to interact with all the agents in the network without cost. When an agent has a complex task to be completed, it simply requests the central manager to seek for the most suitable agents in the network, which could fulfill the task and form coalitions with those agents. This method is not practical or robust, but it can be used as an upper bound of the performance in our experiment.

2. CCF mechanism. This mechanism was proposed by Glinton et al. [12], which does not enable agents to either dynamically adjust their degrees of involvement in a coalition or autonomously join multiple coalitions. These two behaviors, i.e., adjusting degrees of involvement and joining multiple coalitions, are self-organizing behaviors. Thus, it can be conceived that CCF does not integrate self-adaptation. ${ }^{6}$ Through the comparison with CCF, the significance of integrating self-adaptation into coalition formation could be revealed.

3. FCF mechanism. This mechanism is created by us, which is a simplified version of SACF. This mechanism allows agents to breach a contract and leave a coalition by paying penalty to the coalition leader, i.e., Initiator. Agents, however, cannot partially breach a contract. Therefore, agents can join only one coalition at any time step. By comparing with this mechanism, the importance of the notion, i.e., the DoI, can be exposed.

In the agent network, each agent is randomly assigned a single resource $r_{a} \in[1, \varepsilon]$. For simplicity, tasks are created by randomly generating resource requirements $(R(\theta))$. The number of resources required for a given task is chosen

6. Glinton et al. enabled the agent network structure to be changed, namely that each agent can remove its current neighbors and establish connections with other agents as its new neighbors. This agent network structure adaptation process was called self-adaptation in their paper. The aim of their paper is to investigate whether limiting the number of neighbors of each agent has impact on the coalition formation result. For the $C C F$ algorithm itself, i.e., the coalition formation algorithm employed in their paper, no self-adaptation concept was introduced.

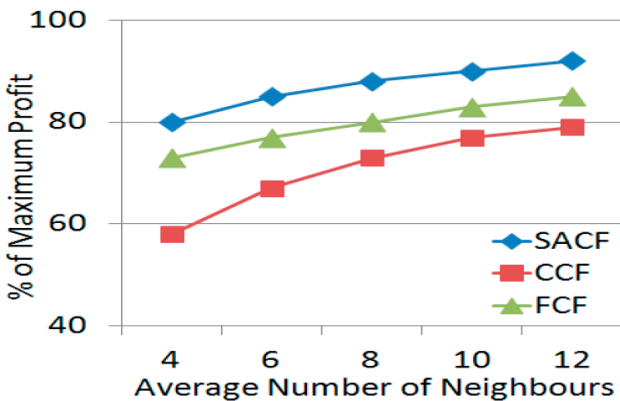

Fig. 1. Profit on different average degrees relative to $C M$.

uniformly from $[1, \varepsilon]$, as a result $|R(\theta)| \leq \varepsilon$. Then, each $r_{\theta}^{l} \in$ $R(\theta)$ is selected randomly from $[1, \varepsilon]$. In addition, at each time step, a task arrives at the agent network with a probability $\lambda$. The required time to complete a task $(P D(\theta))$ is a random positive integer and the latest start time of a task $(D L(\theta))$ is also a random positive integer, which must be greater than the task arrival time $(A T(\theta))$. The task is then randomly assigned to an IDLE agent for allocation as described in Section 3. Finally, the evaluation criteria consist of Profit ${ }_{\text {Net }}$, which is the summation of each individual agent's profit, and time consumed by these mechanisms. Profit $_{\text {Net }}$ can be calculated by using

$$
\text { Profit }_{\text {Net }}=\sum_{i=1}^{|A|}\left(\text { reward }_{i}+\text { recPenalty } y_{i}-\text { penalty }_{i}\right) \text {. }
$$

In (8), each agent's profit is the difference between its income and payout. An agent's, say $a_{i}{ }^{\prime}$ s, income consists of the reward, which is obtained when it completes tasks $\left(\right.$ reward $\left._{i}\right)$, and the penalty, which is received from other agents when they change their degrees of involvement in that agent's coalition (recPenalty $i)$. An agent's payout is the penalty it pays to the coalition leaders, i.e., the Initiators, when it changes its degrees of involvement in their coalitions (penalty $)_{i}$ ). $|A|$ denotes the number of agents in the network. As this paper mainly focuses on theoretical study, we just use a general programming language, i.e., JAVA, to simulate the proposed mechanism. In the experiment, "agent" is initially programmed as a class and then all agents are the objects of this class, and resources are simply represented as integers. Moreover, the experiment is run on Windows XP SP3 operating system with Intel Core 2 Duo $3 \mathrm{GHz}$ CPU and 2 GB RAM. The experimental results are obtained by averaging 100 runs.

For clarity, the values of parameters, which are exploited in these experiments and their meanings are listed in Table 1. These values were chosen experimentally to provide the best results.

\subsection{Experimental Results}

Figs. 1, 2, and 3 demonstrate the relative percentage profits obtained by $S A C F, C C F$, and $F C F$, respectively, compared with the maximum profit, which is gained by the $C M$. It can be seen that $S A C F$ performs consistently better than $C C F$ and FCF in all situations. This is because that $S A C F$ allows an agent to dynamically join more than one coalitions by adapting its DoI in each coalition and this mechanism can potentially make each agent get more profit. 


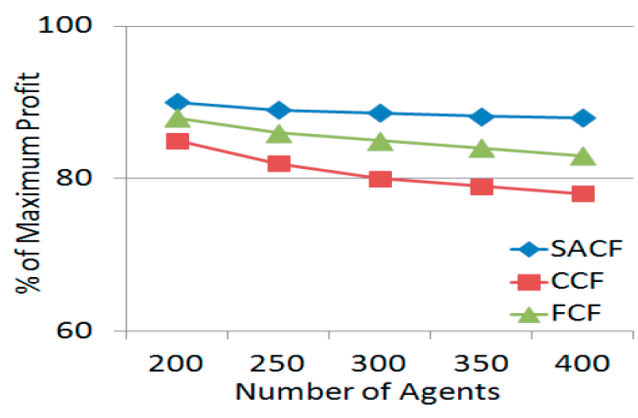

Fig. 2. Profit on different number of agents relative to $C M$.

In Fig. 1, (where the number of agents is 300 and task arrival probability is 0.5 ), with the increasing average number of neighbors, the profits obtained by agents under $S A C F, C C F$, and FCF are gradually ascending. This is because that when each agent has more neighbors, an Initiator can form coalitions for more tasks in a predefined period because it does not always need to commit tasks to other agents. In other words, each agent having more neighbors can accelerate coalition formation process and make more tasks to be completed in the predefined period. It should also be noted in Fig. 1 that the profit obtained by agents under $C C F$ rises much more sharply than that under $S A C F$ and $F C F$, which means that CCF depends heavily on the density (average number of neighbors) of a network.

In Fig. 2, (where the average number of neighbors is 8 and task arrival probability is 0.5 ), when more agents form a network, the profit obtained by agents under CCF decreases steeply but that under both $S A C F$ and FCF keeps relatively steady. This can be explained by the fact that when the number of agents increases but the average number of neighbors of each agent is fixed, each task is potentially to be allocated through a longer commitment chain and thus, in a predefined period, less tasks can be allocated. Under $S A C F$, however, an agent can dynamically join multiple coalitions to execute more than one tasks and, therefore, can obtain extra profit. Likewise, under $F C F$, an agent has the flexibility to leave a coalition and join another coalition. Thereby, this flexibility encourages agents to join more profitable coalitions, and this flexibility can bring extra profits to agents.

In Fig. 3, (where the number of agents is 300 and the average number of neighbors is 8 ), when, at each time step, a task arrives more possibly, the profit obtained by agents under $C C F$ reduces remarkably, while, in comparison, the profit obtained by agents under SACF and FCF drops only a little. This can be ascribed to the fact that when tasks arrive

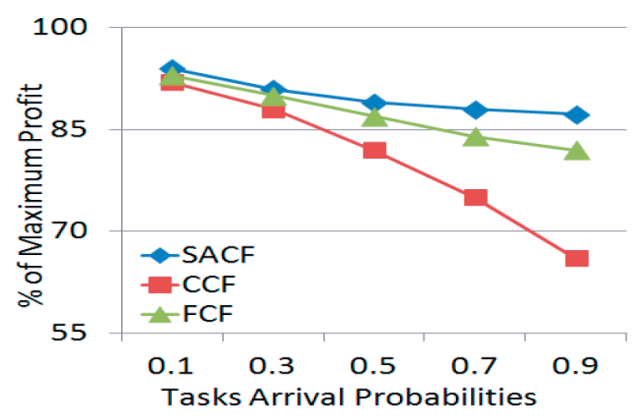

Fig. 3. Profit on different task arrival probabilities relative to $C M$.

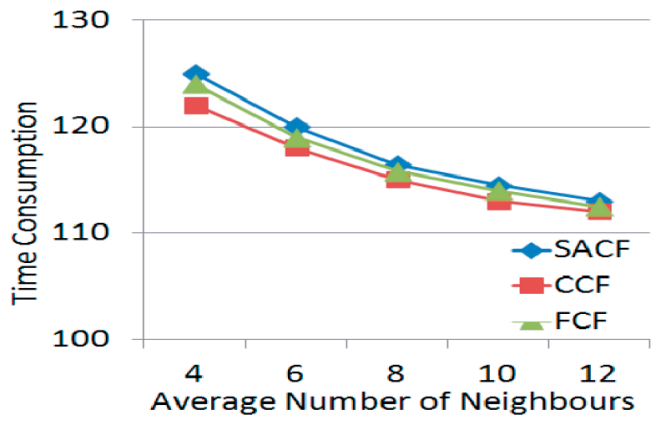

Fig. 4. Time consumption of different mechanisms.

with a low probability, which results in a small number of tasks in the agent network, Initiators relatively have low possibilities to encounter BUSY agents during their task allocation processes, so all the three mechanisms, $S A C F$, $C C F$, and FCF, can obtain decent performance. Nevertheless, once tasks arrive with a very high probability, i.e., a large number of tasks in the network, Initiators are very likely to meet BUSY agents during their task allocation processes. Initiators under the CCF mechanism then have to give up the BUSY agents and try to find other IDLE agents, which decreases the possibility of successful coalition formation. Under $S A C F$ and $F C F$, even if Initiators meet BUSY agents, these Initiators still have the chance to establish coalitions with those BUSY agents. Thus, agents are more possible to achieve more profits. The acute readers might consider that, under the FCF mechanism, once an agent breaches a contract, i.e., leaving a coalition, and joining another coalition, the number of successfully formed coalitions does not increase compared with the CCF mechanism. This consideration is reasonable. However, under the $F C F$ mechanism, when an agent leaves a coalition, it has to pay penalty to the coalition leader, i.e., the Initiator. This penalty is counted as a profit of the Initiator, so the overall profit of the entire agent network still rises.

In Fig. 4, (where the number of agents is 300 and task arrival probability is 0.5 ), the average time consumption of each task is displayed, which exhibits that the three mechanisms spent almost the same time length to finish each simulation run (which consists of 50,000 time steps). This is due to the fact that when an Initiator, under CCF, meets a BUSY agent, the Initiator has to bypass this BUSY agent and tries to find an IDLE agent, but an Initiator, under $S A C F$ and FCF, can also negotiate with the BUSY agent and the BUSY agent may join the Initiator's coalition. Thereby, agents under $S A C F$ and FCF need to negotiate with Initiators regarding their participation and this negotiation obviously costs some extra time. On the other hand, agents under CCF have to bypass some BUSY agents when allocating tasks and bypassing BUSY agents to find suitable IDLE agents also needs some additional time. Furthermore, it can be found that the time consumption of $F C F$ is a little less than that of $S A C F$. This is because that under FCF, when an agent decides to breach a contract, it does not need to adjust its $D o I$ values, since it can join only one coalition. However, under $S A C F$, an agent, which joins a new coalition, has to use Algorithm 4 to adjust the DoI values in its currently participating coalitions. 


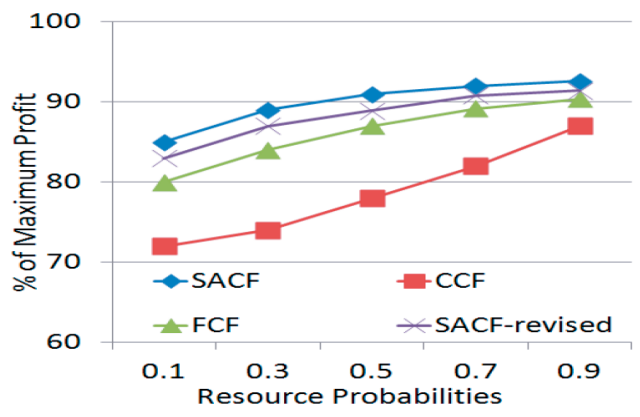

Fig. 5. Profit on different resource probabilities relative to $C M$.

In the previous sections, we assumed that an agent has only one type of resources and an agent cannot leave a coalition once that coalition is formed. Now, we relax these two assumptions and test whether the assumptions have an impact on the performance of the mechanism. To relax the first assumption, we introduce another notion, Resource Probability $(R P)$, in range $(0,1)$, which means that an agent is assigned each type of resources with the probability $R P{ }^{7}$ For the second assumption, we just need to set each $D o I_{l}^{i}=0$, namely that an agent's lower bound of the DoI in each coalition is 0 . This revised $S A C F$ mechanism is recorded as $S A C F$-revised. In addition, the number of agents in the network is set to 300, the average number of neighbors is fixed at 8 and the task arrival probability is 0.5 .

It can be seen from Fig. 5 that $S A C F, S A C F$-revised, and $F C F$ have the same trend of performance that the profit grows steadily with the increase of the RP. However, under $C C F$, the profit soars up steeply with the increase of the RP. This is owing to the fact that the profit obtained by agents under CCF heavily depends on how many resources each agent has. If each agent averagely has more resources, each agent has the potential to complete more subtasks of a task and then the task allocation chain would be shortened, which implies that a coalition for a task is more likely to be successfully formed. Therefore, more profits can be obtained by agents overall. Under SACF, SACF-revised, and $F C F$, the RP does not have much impact on them, because these mechanisms enable an agent to simultaneously join multiple coalitions, which means that an agent's resources can be utilized by several coalitions simultaneously. Specifically, it can be found that the profit obtained by agents under $S A C F$ is consistently greater than that under SACF-revised, which means that restricting agents to leave coalitions is more profitable for the entire network. This is due to the fact that when an agent leaves a coalition, the coalition leader has to find another agent to replace the leaving agent. Nevertheless, it is difficult to find another suitable agent for an uncompleted task. With time elapse, both the benefit of a task and the rest time of completing the task are reducing, so obviously, it is difficult to find an agent to finish a task in a limited time with a relatively low benefit. The Initiator will then lose the benefit of the task, since one of its subtasks cannot be completed, (recall the statement in Section 3.1 that an Initiator can get benefit from the task if and only if all the subtasks of a task are completed).

In summary, the performance of our SACF is around 85-95 percent of the upper bound, i.e., the performance of the $\mathrm{CM}$, and, on average, $10-15$ percent better than $C C F$ with nearly the same time consumption. Compared to the other two revised

7. It has to be guaranteed that an agent has at least one type of resource. version of $S A C F$, i.e., FCF and $S A C F$-revised, $S A C F$ outperforms them as well. This result demonstrates that the effect of incorperating self-adaptation into coalition formation is significant and the assumption, not allowing agents to leave formed coalitions, is reasonable and profitable.

\section{CONCLUSION}

This paper introduced a self-adaptation-based dynamic coalition formation mechanism, which enables agents to dynamically adjust their degrees of involvement in different coalitions to achieve efficient task allocation. The proposed mechanism considers the existence of a neighborhood network structure among agents and integrates the selfadaptation concept. To realize the self-adaptation concept, a negotiation protocol was employed. The integration of selfadaptation results in good performance compared with another famous coalition formation mechanism [12] and other two revised versions of our mechanism. In addition, this paper focuses on using self-adaptation concept to solve coalition formation problem, instead of developing a selfadaptation system. That is why some unexpected behaviors, which are likely to happen in self-adaptation systems, e.g., conflicts among components, did not occur in our design and experimental phases. This research can be exploited in many distributed systems, where resources are distributed and agents are highly autonomous, such as supply chain management, resource management in distributed agent-based grid systems and service-oriented cloud computing, which involve resource discovery, resource negotiation and resource composition.

In the proposed coalition formation mechanism, a negotiation protocol is employed to make contracts between Initiators and Participants. It is conceived that a more efficient negotiation protocol would bring a better result of coalition formation. Thereby, we intend to develop a more efficient negotiation protocol in the future. Additionally, as the main contribution of this paper is the proposed coalition formation mechanism instead of the survey, thorough analysis and comparison with current related studies are not presented. Such a thorough survey is one of our future studies as well.

We are also interested in developing a network reorganization mechanism, also known as network structure adaptation mechanism, to incorporate with the proposed coalition formation mechanism to achieve better results of coalition formation. For example, agent $a_{i}$ connects to agent $a_{j}$, and $a_{j}$ also links with another agent $a_{k}$. If $a_{i}$ often needs help from $a_{k}$, then $a_{i}$ may want to directly connect to $a_{k}$ to save communication overhead and time consumption. On the other hand, if $a_{i}$ rarely communicates with its neighbor $a_{j}$, then $a_{i}$ may want to disconnect with $a_{j}$ to save computation cost associated with taking $a_{j}$ into account whenever $a_{i}$ initializes a coalition formation process. According to this example, it can be found that a network reorganization mechanism is helpful for a coalition formation mechanism to achieve better results compared with the one, which does not use a reorganization mechanism. Finally, we plan to extend this mechanism to an open and mobile agent network, where new agents can join as well as existing agents can leave the network, and agents can also have mobility to travel in the network. To realize this scheme, another network reorganization mechanism is necessary, because whenever new agents join or existing agents leave or agents travel in the network, the network structure is inevitably changed. 


\section{REFERENCES}

[1] C.L. Ortiz, R. Vincent, and B. Morisset, "Task Inference and Distributed Task Management in the Centibots Robotic System," Proc. Fourth Int'l Joint Conf. Autonomous Agents and Multiagent Systems (AAMAS '05), pp. 860-867, July 2005.

[2] V. Lesser, C.L. Ortiz, and M. Tambe, Distributed Sensor Networks: A Multiagent Perspective. Springer, 2003.

[3] D. Pardoe and P. Stone, "An Autonomous Agent for Supply Chain Management," Handbooks in Information Systems Series: Business Computing, vol. 3, pp. 141-173, 2009.

[4] K.M. Sim, "Agent-Based Cloud Computing," IEEE Trans. Services Computing, vol. 5, no. 4, pp. 564-577, Oct.-Dec. 2012.

[5] J. Cao, S.A. Jarvis, S. Saini, D.J. Kerbyson, and G.R. Nudd, “Arms: An Agent-Based Resource Management System for Grid Computing," Scientific Programming, vol. 10, pp. 135-148, 2002.

[6] M. Sims, C.V. Goldman, and V. Lesser, "Self-organization through bottom-up coalition formation," Proc. Int'l Joint Conf. Autonomous Agents and Multiagent Systems (AAMAS '03), pp. 867-874, July 2003.

[7] O. Shehory and S. Kraus, "Methods for Task Allocation via Agent Coalition Formation," Artificial Intelligence, vol. 101, nos. 1/2, pp. 165-200, May 1998.

[8] T. Rahwan, S.D. Ramchurn, A. Giovannucci, and N.R. Jennings, "An Anytime Algorithm for Optimal Coalition Structure Generation," J. Artificial Intelligence Research, vol. 34, pp. 521-567, 2009.

[9] T. Michalak, J. Sroka, T. Rahwan, M. Wooldridge, P. McBurney, and N.R. Jennings, "A Distributed Algorithm for Anytime Coalition Structure Generation," Proc. Int'l Joint Conf. Autonomous Agents and Multiagent Systems (AAMAS '10), pp. 1007-1014, May 2010.

[10] S.D. Ramchurn, M. Polukarov, A. Farinelli, C. Truong, and N.R. Jennings, "Coalition Formation with Spatial and Temporal Constraints," Proc. Int'l Joint Conf. Autonomous Agents and Multiagent Systems (AAMAS '10), pp. 1181-1188, May 2010.

[11] M.E. Gaston and M. desJardins, "Agent-Organized Networks for Dynamic Team Formation," Proc. Int'l Joint Conf. Autonomous Agents and Multiagent Systems (AAMAS '05), pp. 230-237, July 2005.

[12] R. Glinton, K. Sycara, and P. Scerri, "Agent Organized Networks Redux," Proc. 23rd Nat'l Conf. Artificial Intelligence (AAAI '08), pp. 83-88, July 2008.

[13] M.E. Gaston and M. desJardins, "The Effect of Network Structure on Dynamic Team Formation in Multi-Agent Systems," Computational Intelligence, vol. 24, no. 2, pp. 122-157, 2008.

[14] G.D.M. Serugendo and M.-P. Gleizes, "Self-Organisation and Emergence in MAS: An Overview," Informatica, vol. 30, no. 1, pp. 45-54, 2006.

[15] J. Stringer, "Operational Research for 'Multi-Organizations'," Operational Research Quarterly, vol. 18, no. 2, pp. 105-120, 1967.

[16] J.P. Aubin, "Cooperative Fuzzy Games," Math. Operations Research, vol. 6, no. 1, pp. 1-13, 1981.

[17] A. Rubinstein, "Prefect Equilibrium in a Bargaining Model," Econometrica, vol. 50, no. 1, pp. 97-109, 1982.

[18] B. An, V. Lesser, D. Irwin, and M. Zink, "Automated Negotiation with Decommitment for Dynamic Resource Allocation in Cloud Computing," Proc. Int'l Joint Conf. Autonomous Agents and Multiagent Systems (AAMAS '10), pp. 981-988, May 2010.

[19] B. An and V. Lesser, "Negotiation over Decommitment Penalty," Proc. Int'l Joint Conf. Autonomous Agents and Multiagent Systems (AAMAS '11), pp. 1101-1102, May 2011.

[20] B. An, V. Lesser, and K.M. Sim, "Strategic Agents for MultiResource Negotiation," J. Autonomous Agents and Multi-Agent Systems, vol. 23, pp. 114-153, 2011.

[21] P. Faratin, C. Sierra, and N. Jennings, "Negotiation Decision Functions for Autonomous Agents," J. Robotics and Autonomous Systems, vol. 24, nos. 3/4, pp. 159-182, 1998.

[22] J. Dall and M. Christensen, "Random Geometric Graphs," Phys. Rev. E., vol. 66, p. 016121, 2002.

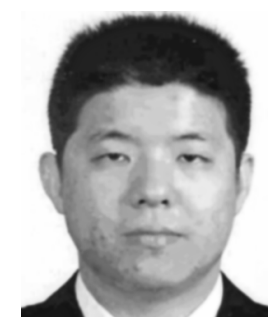

Dayong Ye received the BEng degree from Hefei University of Technology, P.R. China, in 2003 and the MSc degree from the University of Wollongong, Australia, in 2009. Now, he is working toward the $\mathrm{PhD}$ degree in computer science at the University of Wollongong, Australia. His research interests focus on self-organizing multiagent systems and their applications.

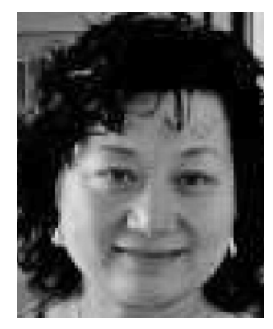

Minjie Zhang received the BSc degree from Fudan University, P.R. China, in 1982 and the $\mathrm{PhD}$ degree in computer science degree from the University of New England, Australia, in 1996. Now, she is an associate professor of computer science at the University of Wollongong, Australia. Her research interests include multiagent systems, agent-based simulation, and modeling in complex domains. She is a member of the IEEE.

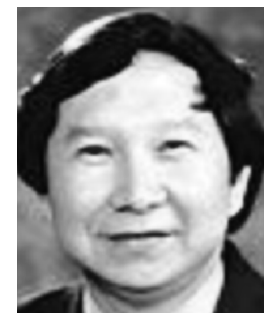

Danny Sutanto received the BEng and $\mathrm{PhD}$ degrees from the University of Western Australia, in 1978 and 1981, respectively. Now, he is a professor of power engineering at the University of Wollongong, Australia. His research interests include energy storage systems and voltage stability. He is a senior member of the IEEE.

For more information on this or any other computing topic, please visit our Digital Library at www.computer.org/publications/dlib. 